\title{
EL GOBIERNO EN LA SOCIEDAD COOPERATIVA COMO BASE DE LA COHESIÓN SOCIAL: EL CASO DE UNA SOCIEDAD COOPERATIVA AGRARIA
}

\author{
POR \\ Cristina PEDROSA ORTEGA ${ }^{1}$ y \\ Elia GARCÍA MARTÍ ${ }^{2}$
}

\section{RESUMEN}

Dadas las características del entorno actual, en el que se ha producido un aumento de la sensibilidad hacia los problemas éticos, se ha globalizado la economía y estamos asistiendo a un aumento de la competencia, se ha producido un cambio en la concepción convencional de la organización.

Como respuesta a la complejidad de la situación actual y para asegurar la supervivencia de la empresa aparece la teoría de los stakeholders. Dicha teoría trata de lograr un buen gobierno a través de la integración de los objetivos de todos los stakeholders de la organización.

Por otra parte, en la cultura de la sociedad cooperativa se refleja como objetivo de este tipo de empresa, el conseguir el desarrollo sostenible de la comunidad en la que se implanta. Esto supone que la cohesión social con su entorno sea considerada como base de la ventaja competitiva de la fórmula cooperativa.

Por ello, en este trabajo partiendo de la búsqueda de un gobierno en la sociedad cooperativa que integre a todos los grupos interesados en esta, vamos a intentar observar

\footnotetext{
${ }^{1}$ Departamento de Organización de Empresas, Marketing y Sociología. Universidad de Jaén. cpedrosa@ujaen.es

${ }^{2}$ Profesora Titular de Universidad. Departamento de Organización de Empresas, Marketing y Sociología. Universidad de Jaén. egarcia@ujaen.es
}

REVESCO N 106 - Tercer Cuatrimestre 2011 - ISSN: 1885-8031 - www.ucm.es/info/revesco

DOI: 10.5209/rev_REVE.2011.v106.37372

Fecha de recepción: 27/09/2010

Fecha de aceptación: 14/03/2011 
cómo se consigue el desarrollo y la cohesión social de la zona en la que se encuentra la organización, siendo esta realidad clave para lograr una ventaja competitiva.

Palabras clave: Cultura cooperativa, Teoría de los Stakeholders, Gobierno Cooperativo, Cohesión Social, Ventaja Competitiva

Claves ECONLIT: M100, M140, M190

\title{
GOVERNANCE IN THE COOPERATIVE AS A BASE OF SOCIAL COHESION: THE CASE OF AN AGRARIAN COOPERATIVE
}

\begin{abstract}
Considering the characteristics of the environment, in which an increase of the sensibility has taken place towards the ethical problems, the economy has been globalized and the competition has increased, a change has taken place in the conventional conception of the organization.
\end{abstract}

To answer to the complexity of the situation and to assure the survival of the company appears the stakeholder's theory. This theory tries to achieve a good government thought the integration of the interest of all the stakeholders of the organization.

On the other hand, in the culture of the cooperative it is reflected like target of this type of company, obtaining the sustainable development of the community in which it is implanted. This supposes that the social cohesion with his environment is considered to be a base of the competitive advantage of the cooperative.

In this paper we search of a government in the cooperative which integrates the objectives of the interested groups, we will try to see how it manages the development and the social cohesion of the area in which the organization is. This could be the key to achieve a competitive advantage.

REVESCO N 106 - Tercer Cuatrimestre 2011 - ISSN: 1885-8031 - www.ucm.es/info/revesco 
Keywords: Cooperative culture, Stakeholder theory, Cooperative Governance, Social cohesion, Competive Advantage

\section{INTRODUCCIÓN}

Nos encontramos en una etapa en la que la sociedad y la economía sufren cambios rápidos y extensos asociados con la globalización, el progreso tecnológico, la volatilidad y las elevadas exigencias de la demanda de los consumidores, la desregularización del mercado, la tendencia a la concentración empresarial de las sociedades, etc. (Martínez, 1990; Menguzzato y Renau, 1991; Brealey y Myers, 1993; Mascareñas y Lejarriaga, 1993; Sanchís, 1995; Arcas y Ruiz, 1997; Hernández y Munuera, 1997; Juliá, 1999; Planells y Mir, 2000). Todos estos cambios han supuesto un fuerte incremento de la competencia a nivel internacional debido a que el entorno competitivo es cada vez más incierto y complejo. Como consecuencia, las características de los mercados actuales hacen que sea cada vez más difícil conseguir una ventaja competitiva y que pueda ser prolongada en el tiempo, por lo que nuevas formas de gestión y de gobierno son necesarias para competir.

En concreto, el buen gobierno puede ser entendido como hacer el bien para todos los implicados en la organización. Por tanto, desde la organización se ha de intentar crear nuevas fórmulas de equilibrio en el gobierno de la empresa, donde todos los afectados por la acción de ésta se encuentren tratados adecuadamente. Así desde esta perspectiva, todas las partes interesadas en la empresa juegan un papel determinante en el comportamiento y consecución del éxito a largo plazo de la organización.

Esta realidad ha sido recogida en los códigos de buen gobierno que representan pronunciamientos en torno al gobierno corporativo con recomendaciones precisas y de adopción voluntaria para las empresas. Estos códigos suelen ser elaborados por comisiones especializadas de expertos, con frecuencia constituidas a iniciativa de organismos oficiales, con el cometido fundamental de estudiar los criterios y pautas a que deben someterse las sociedades en un contexto determinado, y pronunciarse con una serie de recomendaciones que mejoren el comportamiento de las organizaciones respecto a la transparencia informativa, composición y funcionamiento de los órganos de gobierno y relación con los distintos grupos de interés (AECA, 2004). 
Entre los informes y códigos elaborados en los últimos tiempos sobre el gobierno societario hay que destacar el informe de la OCDE del año 2004. En este informe se recoge en el preámbulo la siguiente afirmación: "El gobierno corporativo comprende una serie de relaciones entre la dirección de la empresa, su consejo de administración, sus accionistas y otros grupos de interés". En concreto cita a inversores institucionales, accionistas individuales, entidades de crédito, empleados, gobiernos y otras partes interesadas, todos ellos juegan un papel determinante en el comportamiento y consecución del éxito a largo plazo de la empresa (Lizcano, 2006).

En concreto en el cuarto principio de los seis emitidos por la OCDE (2004), se hace referencia a la importancia de los stakeholders en el gobierno corporativo: "El marco de gobierno corporativo debe reconocer los derechos de los grupos de interés establecidos legalmente o acordados mutuamente y promover activamente la cooperación entre las empresas y dichos grupos para la creación de riqueza, trabajo y la sostenibilidad de empresas financieramente sanas".

"El gobierno corporativo también es responsable de la búsqueda de fórmulas para la efectiva implicación de los grupos de interés en alcanzar el óptimo valor económico de la inversión en capital humano y físico de la compañía. La competitividad y el éxito de la empresa es resultado del trabajo en equipo y de recursos procedentes de distintos grupos de interés: inversores, empleados, entidades de crédito y proveedores. Las empresas deben reconocer que las aportaciones de los distintos grupos de interés constituyen un valor esencial para ser competitivas y rentables. Es por esto por lo que en interés a largo plazo de las compañías es muy importante establecer buenas relaciones con los grupos de interés para la generación de riqueza. El marco de gobierno corporativo debe reconocer que los intereses de la empresa pasan por el reconocimiento de los intereses de los grupos de interés y su contribución al éxito de la compañía a largo plazo" (OCDE, 2004).

Por tanto, en este informe se reconoce explícitamente la aportación a la generación de valor y a la competitividad procedente de los distintos grupos de interés y, en consecuencia, se considera que es responsabilidad del gobierno corporativo la relación con dichos grupos para alcanzar de manera eficaz los objetivos marcados por la empresa. Este informe ha 
supuesto un punto de partida para optar por una perspectiva pluralista en el ámbito del gobierno corporativo incorporando la acepción de empresa socialmente responsable.

Como estamos poniendo de manifiesto, la preocupación actual en relación con el buen gobierno es generalizada (Spear, 2004). Muestra de ello son las diversas publicaciones que han visto la luz en la actualidad, centradas en la temática del gobierno cooperativo (Hermes et al., 2006; Berglöf y Claessens, 2006; Reaz y Hossain, 2007; Taliento, 2007, etc.). Además, el estudio del buen gobierno no se ha realizado solo desde una perspectiva general, sino que otros autores han enfocado sus investigaciones en mercados o códigos de buen gobierno concretos (Mallin, 2003; Pass, 2006; Cheung et al., 2007; McGunable, 2007; Tipuric, et al. 2007; Okike, 2007; Von Rosen, 2007).

Las sociedades cooperativas agrarias, han tenido que responder a este nuevo entorno económico desarrollando innovaciones institucionales y financieras, y adoptando nuevas estrategias en la mayoría de los ámbitos empresariales, en particular, en el de recursos humanos y gobierno. En definitiva, este tipo de entidades intentan responder al entorno en el que se desarrollan, procurando lograr una actuación eficaz que les suponga convertirse en una fórmula empresarial exitosa y lograr una ventaja competitiva (Ruíz et al., 2008).

El propósito de estas organizaciones se sustenta en unos valores y principios específicos que hace que las sociedades cooperativas sean empresas con rasgos distintivos de las empresas capitalistas, lo que las puede situar en posición de ventaja competitiva frente a otras empresas y que puede ser el centro de su éxito y de su supervivencia.

Así, existe un creciente interés en el estudio de las sociedades cooperativas agrarias, debido a la importancia de este tipo de organizaciones para el tejido empresarial de cualquier país en el mundo pues "el cooperativismo agrario se caracteriza por ser el principal dinamizador económico y social de las zonas rurales, porque crea empleo, fija la población en su territorio, incrementa el tejido empresarial, genera riqueza, mejora el bienestar social de los ciudadanos y contribuye a la conservación de paisajes y recursos naturales ${ }^{3}$ ".

\footnotetext{
3 Anuario de la Economía Social 2007-2008 de la Confederación de Empresarial Española de la Economía Social.
} 
En España contamos con aproximadamente unas 4.000 empresas cooperativas agrarias que suponen una parte muy importante de la actividad económica del sector agroalimentario español, con una facturación en 2008 de 18,9 millones de euros. Además, concentran una parte significativa del sector agrario español, dando empleo a más de 73.800 trabajadores, la mayor parte en el medio rural, lo que convierte a este tipo de empresas en un auténtico motor económico, social y cultural de las zonas rurales y menos pobladas de nuestro país (CCAE, $2011)^{4}$

En definitiva, las empresas en general y las sociedades cooperativas en particular, han de adaptar su modelo de gestión a las nuevas necesidades del entorno actual si pretenden seguir compitiendo en el mercado. Consideramos que para desarrollar un modelo de management efectivo en las sociedades cooperativas se han de tener en cuenta, los intereses de todos los stakeholders que las integran, incorporando sus objetivos y exigencias. Para ello es necesario que el gobierno de la sociedad cooperativa tenga como base la integración de todos sus grupos de interés, pues el papel que ocupan los stakeholders en las organizaciones va tomando cada vez mayor importancia. Las empresas han de estar preparadas para identificarlos, así como para establecer sus necesidades y expectativas en relación con la organización, con el objetivo de lograr un alineamiento entre los intereses de estos grupos y los de la propia sociedad cooperativa. Este alineamiento de intereses consiste en compatibilizar los objetivos de todos los stakeholders de la empresa con el fin de que se puedan alcanzar las expectativas de todos ellos en los niveles deseados y no aparezcan conflictos. Siguiendo a Guerras y Navas (2007), el alineamiento de intereses se conseguiría mediante la negociación de todos los stakeholders hasta llegar al equilibrio, fijando un objetivo que, en cierto modo, integra a los de todos los grupos.

En este trabajo se va a tratar de explicar cómo, a partir de la consecución de un buen gobierno en la organización, se integra a todos los grupos de interés en la empresa y se posibilita el desarrollo de la zona y la cohesión social de la misma. La escasez de información en este ámbito y la opción de analizar cómo el buen gobierno de la empresa facilitaba la cohesión social de la zona en su contexto, nos han llevado a desarrollar un estudio de carácter cualitativo a partir del análisis de un caso.

\footnotetext{
${ }^{4}$ www.agro-alimentarias.coop
} 
La estructura del presente trabajo parte de una introducción, después muestra la necesidad y realidad del buen gobierno cooperativo incluyendo la teoría que desarrolla un modelo de buen gobierno, para más adelante estudiar cómo a partir de la búsqueda de la cohesión social la sociedad cooperativa logra conseguir una ventaja competitiva en el mercado. Después se muestra un caso de estudio en el que se recoge está realidad para terminar con las conclusiones del trabajo.

\section{EL GOBIERNO EN LA SOCIEDAD COOPERATIVA AGRARIA}

El gobierno democrático es un rasgo característico de las sociedades cooperativas en general, que se manifiesta en la participación de los socios y de otros grupos en la vida cooperativa. Esta participación es un deber que se deriva de los derechos que la legislación atribuye a los socios como empresarios-propietarios y se canaliza a través de los órganos de la sociedad cooperativa (Hernández, 2001).

Ahora bien, ¿influye el gobierno democrático de las sociedades cooperativas en la consecución del éxito empresarial de este tipo de organizaciones? Ésta es una cuestión de central importancia para la investigación pues consideramos que la sociedad cooperativa que se caracterice por sus prácticas de buen gobierno, que incluye a todos los grupos de interés de la empresa, obtendrá una ventaja competitiva que la diferenciará de las demás entidades, asegurando así su continuidad en el mercado.

Siguiendo a Hernández et al. (2008), definimos gobierno cooperativo como “(...) la forma de administrar, dirigir y guiar a la sociedad cooperativa hacia el logro de sus objetivos", por tanto, su finalidad es la de guiar a sus directivos mediante mecanismos institucionales democráticos para que su gestión sea en beneficio de sus socios y, adicionalmente, de las comunidades donde estos viven (Chaves y Soler, 2004). Atendiendo a Davis (2001), el gobierno cooperativo tiene, como una parte central de su objetivo principal, el mantenimiento de identidad cooperativa. Este objetivo incluye la entrega eficiente de servicios y productos demandados por los socios de la cooperativa en el contexto de construir su comunidad común. Y como afirman Romero y Pérez (2003), existe un alto grado de 
asociación entre la participación en el gobierno de la sociedad cooperativa y el desarrollo en la misma de una cultura organizacional apropiada.

Así, las características del gobierno cooperativo se presentan como un elemento diferenciador de las empresas de economía social frente a las empresas capitalistas convencionales. En este tipo de empresa la gestión es ejercida directamente por los socios a través de la asamblea general, y son éstos los que participan en la toma de decisiones y en la fijación de sus políticas de actuación. Se intenta responsabilizar al socio de su propio futuro, implicándole en el destino de la organización.

De este modo, podemos afirmar que las sociedades cooperativas ante todo son, sociedades de personas en las que los socios pueden gobernar mediante dos tipos de instrumentos: indirectos (participación del socio en la actividad de la sociedad cooperativa); y directos (participación en la organización interna, en la elección de los administradores y cargos directivos, control de la gestión, etc.), siempre bajo el cumplimiento y el respeto a los principios cooperativos establecidos en la Alianza Cooperativa Internacional en Manchester (1995). Además, para hacer frente a los desafíos del entorno, las sociedades cooperativas han tenido que incorporar personas cualificadas, que son los directivos profesionales, con el objetivo de que la empresa pueda seguir siendo competitiva en el mercado, logrando la supervivencia y la consecución del éxito empresarial (Pedrosa, 2009).

Por tanto, en las empresas cooperativas, la incorporación de directivos profesionales constituye un elemento transformador de su lógica de gobierno. Según Cornforth (2002), la perspectiva democrática de gobierno sugiere que la tarea de estos directivos profesionales es representar los intereses de los socios en la cooperativa. Nos podemos preguntar, ¿a qué se debe la incorporación de este tipo de stakeholder en el gobierno de la sociedad cooperativa? Pues bien, según este mismo autor, la presencia de esta figura es debida a una serie de factores tales como: la creciente apatía de los socios en la participación de la toma de decisiones de la empresa; la pérdida de la identidad cooperativa; la mayor dimensión de la sociedad cooperativa; y la profesionalización de estos directivos entre otros.

Como solución a todos estos problemas a los que se enfrenta el gobierno de la sociedad cooperativa, se plantea la necesidad de la búsqueda y consecución de un modelo de 
buen gobierno cooperativo que, en palabras de Chaves (2004), “es aquel que logra el máximo nivel de desempeño económico de la sociedad cooperativa compatible con la preservación o desarrollo de su identidad cooperativa".

El buen gobierno en las sociedades cooperativas se plantea más como un problema de cultura de dirección que un problema de estructuras democráticas, es un tema del que se afirma "es más fácil hablar y escribir, que alcanzar". Para la mejora del gobierno cooperativo se deberían seguir las siguientes premisas según afirma Davis (2001):

- Un buen gobierno es ante todo la defensa de la identidad cooperativa y sus objetivos.

- Un buen gobierno requiere el ejercicio de valores de liderazgo.

- Un buen gobierno requiere una cultura cooperativa que satisfaga las necesidades de sus socios y de los demás stakeholders.

\subsection{La teoría de los stakeholders en el modelo cooperativo}

En la búsqueda del buen gobierno cooperativo la teoría de los stakeholders permite tener una visión más amplia de las relaciones que se establecen en la organización y las responsabilidades que ello conlleva, más allá de la maximización del beneficio, e incluye los intereses y la demanda de entidades diferentes a los accionistas (Mitchell et al., 1997), es decir, se centra en que las empresas deben ser responsables ante un conjunto de grupos interesados en ella, y no solamente preocuparse por los socios (Cornforth, 2002; Spear, 2004).

Por ello, el origen de la teoría de los stakeholders se explica partiendo de que la organización ha pasado de considerarse como un sistema de transformación de los recursos procedentes del mercado en busca de la obtención de un producto final, y en orden a obtener un beneficio máximo, a considerar, además, un conjunto de grupos que razonan y esperan de las actuaciones de ésta y ante los cuales siente responsabilidad (Caballero, 2005).

Así y, siguiendo a Caballero (2005), podemos diferenciar tres etapas en el desarrollo del término stakeholder: (1) una primera etapa inicial, de Stanford Research Institute (SRI, 1963), que considera a los stakeholders como el eje de la supervivencia de la organización; (2) a continuación nos encontraríamos con la etapa decisiva del término, que sería la aportada por Freeman en 1984 y que lo relaciona con la disciplina de Dirección Estratégica; (3) por 
último, diferenciamos también la etapa actual, en la que las relaciones entre la organización y cada uno de los stakeholders han pasado de ser una actividad de escasa importancia en el éxito competitivo a considerarse una necesidad central, dado que la empresa ha pasado de considerar a los stakeholders de los que necesita para sobrevivir a considerar a todos aquellos que tienen algún interés o se sienten afectados por aquella, dado que el deseo del alcance conjunto de todos los intereses es necesario en el desarrollo y control de las estrategias de la organización, buscando el equilibrio oportuno de todos ellos.

La teoría de los stakeholders se considera reciente dado que, aunque se remonta al siglo pasado, es a finales de los 80 cuando comienzan a destacar significativas aportaciones que ocupan hasta nuestros días, pues es Freeman (1984) el que se puede considerar su precursor. En resumen, la teoría de los stakeholders supone una visión integral de las organizaciones, al pretender conseguir un equilibrio entre los stakeholders de la empresa para poder adaptarse a los continuos cambios que se producen en su entorno y conseguir la supervivencia en el tiempo (Pedrosa, 2009).

Se trata de dar el protagonismo necesario a las personas y sus intereses dado que son las que, en última instancia, marcan las pautas del progreso económico real de la organización (Álvarez, 1999). Esta posición se puede utilizar como una metodología de gestión empresarial del ámbito estratégico de la organización y que permitirá a las sociedades cooperativas agrarias alcanzar el éxito en el seno de su localidad.

Como consecuencia de lo anterior, la sociedad cooperativa tiene como objetivo principal la identificación de los stakeholders, pues para que pueda ser efectiva, ha de ser capaz de encontrar el equilibrio entre los intereses de todos sus stakeholders y los suyos propios. Así, las empresas en general se esfuerzan por identificar, conocer y comunicarse con cada uno de ellos, decidiendo el nivel de responsabilidad con cada uno, utilizando un rango cada vez más amplio de indicadores de cumplimiento de resultados que los relacionan con los intereses de todos los grupos (Wheeler y Sillanpaa, 1998).

De este modo y como venimos indicando a lo largo de este trabajo, la sociedad cooperativa agraria que identifique y tenga en cuenta el cumplimiento de los objetivos de todos sus stakeholders como base de un buen gobierno cooperativo, obtendrá una ventaja 
competitiva que la diferenciará del resto y ello supondrá una posición ventajosa en el mercado que le asegurará su supervivencia, alcanzando así el éxito empresarial.

Es vital el saber conciliar todos los intereses y saber hacer llegar como propios cada uno de los flujos a cada uno de los grupos, para que se cumplan los objetivos generales de la empresa. Así se logra la credibilidad de los stakeholders en la organización y la buena imagen de ésta, es decir, la identidad organizacional se verifica en la medida en que los stakeholders ven realizadas sus expectativas en la organización (Scott y Lane, 2000).

\section{BUSCANDO LA COHESIÓN SOCIAL Y LA VENTAJA COMPETITIVA DE LA EMPRESA}

Tras estudiar el buen gobierno nos centramos en la identidad cooperativa como un factor que puede incidir en la supervivencia de la propia cooperativa. Si todos los stakeholders tienen sentimiento de pertenencia a la organización, ésta seguirá siendo competitiva en el mercado, pudiendo llegar a alcanzar el éxito empresarial. Para las sociedades cooperativas la clave del buen gobierno está en reconocer la esencia de la forma cooperativa de empresa y no tratar de imitar a las empresas capitalistas convencionales.

La identidad cooperativa se basa en sus principios cooperativos. Estos constituyen un elemento diferencial que puede proporcionar ciertas ventajas y permitir que las cooperativas partan de una situación favorable para hacer frente a los retos generados por los rápidos e incesantes cambios del entorno que influyen en la competitividad de la cooperativa (Vargas, 1995; 1999; Díaz, 2000; 2004; Agirre, 2001;). No obstante, la influencia de algunos principios es más directa que la de otros (Gómez y Miranda, 2006).

De este modo, las sociedades cooperativas se caracterizan por sus valores, como la democracia, el interés social y la justicia distributiva, que se traducen en unas reglas de funcionamiento determinadas. Este funcionamiento se basa en los denominados valores cooperativos (autoayuda, auto-responsabilidad, democracia, igualdad, equidad y solidaridad). Además, estos valores han de estar acompañados y reforzados por un conjunto de valores éticos que los cooperativistas deben hacer suyos, refiriéndose a la honestidad (honradez), la transparencia (franqueza), la responsabilidad (social y mutua) y la vocación social (interés o 
preocupación por los demás) (García, 2000). Estos valores se materializan en los principios cooperativos que conforman las señas de identidad y los parámetros del gobierno en estas empresas. Es justo reconocer que nos estamos refiriendo a un conjunto de valores que no son en modo alguno monopolio de las sociedades cooperativas. La situación resultante de que a estos valores se les considere cooperativos, es que producen un efecto positivo en los esfuerzos de las personas a la hora de participar en la gestión de una empresa para beneficio de todos sus miembros (Nilsson, 1996).

Así y siguiendo a García (2000), para que una organización de cualquier tipo sobreviva y tenga éxito, debe existir un adecuado conjunto de principios que sirvan de pauta o guía para la acción, sobre los que se basen todas sus políticas y actividades y sirvan para poner en práctica los valores de sus socios. Estos valores se ponen en práctica a través de los principios cooperativos establecidos por la Alianza Cooperativa Internacional (ACI, 1995), que determinan las pautas de actuación de las cooperativas. Estos principios son los siguientes:

1) Adhesión voluntaria y abierta

2) Gestión democrática por parte de los socios

3) Participación económica de los socios inspirada en los valores cooperativos

4) Autonomía e independencia

5) Educación, formación e información

6) Cooperación entre cooperativas

7) Interés por la comunidad

El último principio, objetivo de nuestro trabajo, de interés por la comunidad, hace referencia a que, al mismo tiempo que las sociedades cooperativas se centran en las necesidades y los deseos de sus socios, también trabajan para conseguir el desarrollo sostenible de sus comunidades según los criterios aprobados por los socios. Atendiendo a Moreira (1999), desde el punto de vista de los valores o principios cooperativos, la cohesión social forma parte de la ventaja cooperativa. La esencia del cooperativismo se concentra en los valores de la cooperación y de la solidaridad, en oposición a los valores actuales de individualismo y competición, característicos de la globalización, siendo aquellos valores intangibles y constituyentes de una incontestable ventaja cooperativa (Jordao y Briz, 2004). 
Como se ha visto en el trabajo, los principios cooperativos constituyen los pilares sobre los cuales se estructuran las sociedades cooperativas. Son una guía para las acciones económicas y sociales, creando una cultura empresarial fuerte, que representa una ventaja competitiva frente a otras empresas con culturas débiles y/o variables en el tiempo. De este modo, los principios cooperativos en su conjunto son la fuerza vital de la empresa cooperativa y la puesta en práctica de los valores que conformarán su cultura organizacional. Los principios establecen las estructuras y determinan las actitudes que le dan su perspectiva distintiva. Son las directrices según las cuales los cooperativistas procuran desarrollar sus organizaciones (Tous y Ciruela, 2005).

En los valores y principios presentes en la cultura cooperativa se pone de manifiesto de manera implícita el compromiso con los grupos de interés. Teniendo muy en cuenta las expectativas y necesidades de las personas y la sociedad con la que se relacionan, lo que puede suponer una importante fuente de ventajas competitivas (Tous y Ciruela, 2005). Teniendo en cuenta todos los argumentos expuestos podemos formular la siguiente proposición:

Proposición 1: La consecución del buen gobierno cooperativo a través de la integración de todos los grupos de interés posibilita la cohesión social de la zona en la que implanta la sociedad cooperativa y la consecución de una ventaja competitiva para la empresa.

\section{CASO DE ESTUDIO}

Para justificar la proposición planteada hemos utilizado el estudio de un caso de tipo ilustrativo, siguiendo la metodología de casos establecida por Ryan, Scapens y Teobal (1992). Estos autores nos muestran que los casos ilustrativos intentan mostrar prácticas innovadoras que afectan al desarrollo organizativo de las empresas. La investigación empírica que se realiza en este tipo de casos centra su atención en la descripción del proceso de implantación de las mejores prácticas en el seno de las organizaciones. 
Consideramos está metodología adecuada para la realización de este trabajo, pues nos permite analizar el fenómeno objeto de estudio en su contexto real, utilizando múltiples fuentes de evidencia, cuantitativas y/o cualitativas simultáneamente (Villareal y Landeta, 2007; Yin, 1989, y Eisenhardt, 1989). En particular, se realizaron entrevistas individuales, se revisaron documentos y artículos sobre la empresa analizada y se obtuvo información directa a través de las visitas a la zona donde tiene su sede la empresa objeto de estudio.

El caso elegido, dadas las características del trabajo, tenía que ser una sociedad cooperativa de gran dimensión con una posición consolidada y ventajosa en el mercado. Además, se tenía que caracterizar por sus buenas prácticas de gestión, cumpliendo los principios cooperativos como un modo de conseguir el éxito empresarial.

La empresa elegida ha sido Hojiblanca Sociedad Cooperativa Andaluza por situarse en el puesto número 593 según el ranking de las 5000 mayores empresas de España (Alimarket, 2008) con una facturación ${ }^{5}$ de 291,43 millones de euros en 2008. Esta empresa es el resultado de la unión de un conjunto de cooperativas de primer grado dedicadas a la fabricación de aceite de oliva virgen y aceitunas de mesa. Son 35.000 familias de olivareros ${ }^{6}$ que cultivan 30 millones de olivos en 300.000 hectáreas de olivar. Producen una media anual de 150.000 toneladas de aceite de oliva virgen y 44.000 toneladas de aceitunas de mesa. Estas cifras convierten a esta empresa en el mayor productor mundial de aceite de oliva virgen y aceitunas de mesa y en el primer envasador de aceite virgen extra del país.

A continuación se muestran las características de los stakeholders entrevistados y la ficha técnica de las entrevistas realizadas.

Tabla 1: Características de los stakeholders entrevistados

\begin{tabular}{|l|c|c|c|}
\hline & $\begin{array}{c}\text { Tipo de } \\
\text { stakeholder }\end{array}$ & Edad & $\begin{array}{c}\text { Implicación con la } \\
\text { soc. coop. agraria } \\
\text { de } \mathbf{2}^{\mathbf{0}} \text { grado }\end{array}$ \\
\hline Entrevistado 1 & Directivo & 43 & Director de \\
\hline
\end{tabular}

\footnotetext{
${ }^{5}$ Datos extraídos de la documentación proporcionada por los entrevistados, así como del Informe Anual del Grupo Hojiblanca relativo al año 2007.

${ }^{6}$ Datos recogidos en la página web del Grupo Hojiblanca www.hojiblanca.coop y www.hojiblanca.es
} 


\begin{tabular}{|l|c|c|c|}
\hline & profesional & & comunicación \\
\hline Entrevistado 2 & Socio fundador & 58 & Socio \\
\hline Entrevistado 3 & $\begin{array}{c}\text { Asociación } \\
\text { empresarial }\end{array}$ & 40 & Persona del entorno \\
\hline Entrevistado 4 & $\begin{array}{c}\text { Directivo } \\
\text { profesional }\end{array}$ & 56 & Director general \\
\hline Entrevistado 5 & $\begin{array}{c}\text { Miembro de la } \\
\text { comunidad }\end{array}$ & 50 & Persona del entorno \\
\hline Entrevistado 6 & Socio & 55 & Socio \\
\hline
\end{tabular}

Fuente: Elaboración propia

Tabla 2: Ficha Técnica del estudio del caso de Hojiblanca

\begin{tabular}{|c|c|}
\hline Metodología de investigación & $\begin{array}{l}\text { Estudio de caso único de carácter holístico } \\
\text { (unidad de análisis simple). Estudio } \\
\text { exploratorio, ilustrativo y potencialmente } \\
\text { explicativo }\end{array}$ \\
\hline Unidad de análisis & $\begin{array}{lll}\text { Hojiblanca } & \text { Sociedad } & \text { Cooperativa } \\
\text { Andaluza } & & \end{array}$ \\
\hline Ámbito geográfico & Antequera (Málaga, Andalucía) \\
\hline Universo & $\begin{array}{l}\text { Sociedades cooperativas agrarias de } \\
\text { segundo grado }\end{array}$ \\
\hline Métodos de recogida de la evidencia & $\begin{array}{l}\text {-Revisión documental (documentación y } \\
\text { archivos) } \\
\text {-Realización de entrevistas presenciales en } \\
\text { profundidad; abiertas y semiestructuradas } \\
\text {-Observación directa }\end{array}$ \\
\hline Fuentes de información & $\begin{array}{l}\text { Interna: documentación (memorias, } \\
\text { informes y estudios internos), archivos } \\
\text { (páginas Webs, archivos de } \\
\text { presentaciones, archivos de imagen y } \\
\text { sonido), entrevistas en profundidad, y } \\
\text { contexto físico real. } \\
\text { Externa: publicaciones especializadas, }\end{array}$ \\
\hline
\end{tabular}

REVESCO No 106 - Tercer Cuatrimestre 2011 - ISSN: 1885-8031 - www.ucm.es/info/revesco 


\begin{tabular}{|c|c|}
\hline & $\begin{array}{l}\text { bases de datos, informes de organismos } \\
\text { oficiales y medios de comunicación. }\end{array}$ \\
\hline Informadores clave & $\begin{array}{l}\text { Stakeholders vinculados a nuestra unidad } \\
\text { de análisis }\end{array}$ \\
\hline Métodos de análisis de la evidencia & $\begin{array}{l}\text { Fundamentalmente de tipo cualitativo: } \\
\text {-Identificación y clasificación estructural } \\
\text { de dimensiones clave } \\
\text {-Búsqueda de factores explicativos clave } \\
\text { (proposiciones teóricas) } \\
\text {-Búsqueda de patrón de comportamiento } \\
\text { común (proposiciones teóricas) } \\
\text {-Creación de explicación teórica } \\
\text { (comparación sistemática teórica) } \\
\text {-Identificación de relaciones causales (si } \\
\text { es posible) } \\
\text { - Análisis de decisiones críticas }\end{array}$ \\
\hline Enfoque científico & $\begin{array}{l}\text { Procesos deductivos en la medida que se } \\
\text { parte de proposiciones teóricas de la } \\
\text { revisión de teorías }\end{array}$ \\
\hline $\begin{array}{l}\text { Evaluación del rigor } \quad \text { y } \\
\text { metodológica }\end{array}$ & $\begin{array}{l}\text { Validez (constructiva, interna y externa), } \\
\text { fiabilidad, consistencia (contextual y } \\
\text { teórico - interpretativa) }\end{array}$ \\
\hline Fecha de realización & $\begin{array}{l}\text { Fecha inicio: Abril } 2008 \\
\text { Fecha finalización: Octubre } 2009\end{array}$ \\
\hline
\end{tabular}

Fuente: Adaptado de Villarreal y Landeta (2007)

Con carácter general, la aportación de las sociedades cooperativas se ha puesto de manifiesto desde la Unión Europea, al reconocer el papel desempeñado por el sector cooperativo en el desarrollo territorial, contribuyendo a una mejor integración y cohesión social. Así las sociedades cooperativas se convierten en una tipología empresarial idónea para conseguir objetivos en materia de desarrollo. La participación de los socios en todos sus procesos, el cumplimiento de los principios cooperativos y el asentamiento de estas empresas en zonas rurales son las claves para reconocerles un papel básico en el desarrollo de los 
territorios. Las sociedades cooperativas juegan un papel importante porque representan los intereses de los grupos de la zona en la que se desarrollan y suponen una vinculación con el tejido económico y social de la zona que intenta procurar la creación de empleo, la movilización de recursos, la generación de inversiones y una transmisión de la información para que se desarrollen los procesos de integración y cohesión social (Bel y Ausín, 2007).

La sociedad cooperativa, objeto de nuestro trabajo, representa desde sus inicios a una organización capaz de articular y defender las necesidades de los sectores más débiles de la población. Ésta está al servicio de la persona, cada individuo, sea cual sea el grado de la organización en la que se inserte, no puede apartarse demasiado de la comunidad en la que está inmersa. Por lo que Hojiblanca S. C. A. no ha podido situarse con independencia de la actividad que llevan a cabo sus socios, sino que ha estado y está situada junto a ellos, trabajando por el desarrollo económico y social del entorno en el que se encuentra.

Centrándonos en el entorno y la comunidad local como stakeholder a tener en cuenta para lograr la cohesión social con el entorno de la empresa y asegurar así el éxito de Hojiblanca S. C. A. diremos que garantiza a esta sociedad cooperativa agraria el derecho de construir sus estructuras y, en ocasiones le brinda facilidades para lograrlo: a cambio la comunidad se beneficia de las tasas impositivas por actividades empresariales y por la contribución económica (es una fuente de ingresos) y social de la cooperativa. Además, también tiene intereses depositados en la misma, que trata de defender e intenta que se respeten. Estos intereses incluyen la lucha por el mantenimiento del medio ambiente (no contaminación, evitar ruidos, evitar olores,...), la creación de empleo y disminución de costes sociales, el mantenimiento de la estabilidad social de la comunidad, la formación de polígonos industriales, el desarrollo local, etc. Los conflictos más importante que se podrían producir entre la sociedad cooperativa y la comunidad como stakeholder serían el cierre de plantas y la contaminación atmosférica.

Además, cuando identificamos el entorno como stakeholder nos referimos al entorno natural pues, aunque por su naturaleza no debería ser incluido dentro de la clasificación de los stakeholders, es importante considerarlo como tal porque dichos stakeholders pueden cambiar drásticamente su relación con la sociedad cooperativa en función del trato otorgado por ésta a la naturaleza, el respeto y conservación que se demuestre por dicho entorno. 
Otras manifestaciones de las relaciones de interdependencia que Hojiblanca S. C. A. mantiene con su entorno serían las siguientes: en primer lugar, podemos ver cómo la comunidad y el entorno favorecen el desarrollo de una cultura cooperativa, lo que constituye un aspecto favorable para la continuidad de esta empresa, pues cuenta con el apoyo de su comunidad y a la vez, está favorecida por el entorno en el que desarrolla su actividad.

El valor que es capaz de generar esta sociedad cooperativa tiene tanta importancia para sus socios como para el entorno en que se ubica. En la mayoría de las ocasiones, sobre todo en las localidades de pequeña dimensión, las almazaras cooperativas asociadas a Hojiblanca S. C. A. son el motor de desarrollo y la base de la economía local. Así, el mayor interés por la comunidad asegura un desarrollo y prosperidad de la zona geográfica en la que se implanta la organización. Esta sociedad cooperativa no se aísla de la actividad que llevan a cabo sus socios, sino que por el contrario se sitúa junto a ellos trabajando por la mejora del nivel de vida del entorno en el que se encuentra. Se intenta asegurar la identificación de ideas y necesidades de los miembros de la empresa para fomentar la supervivencia y el éxito de la misma.

Se trata de buscar el desarrollo sostenible de la comunidad en la que está insertada esta sociedad cooperativa, mediante políticas apoyadas por sus socios. Así, en la medida que intervengan más individuos en la organización, será más fácil identificar nuevas ideas y ayudar al desarrollo del entorno. De esta manera, Hojiblanca S. C. A. puede ser considerada como una asociación democrática de emprendedores.

En resumen, podemos decir que el principio interés por la comunidad hace referencia al grado de relación o unión con lo local de toda experiencia cooperativa. El componente endógeno es considerado como un elemento de gran importancia tanto para el funcionamiento como para el desarrollo de las cooperativas (Moreira, 1999). Desde el punto de vista de los valores o principios cooperativos, la cohesión social forma parte de lo que este autor denomina la ventaja cooperativa. 
Asimismo, creemos que el alineamiento entre los socios y los directivos profesionales en el cumplimiento de los principios y valores cooperativos en la sociedad cooperativa favorece la consecución del éxito empresarial y la ventaja competitiva de la organización.

Siguiendo con el caso de estudio analizado hemos observado la importancia que tiene Hojiblanca Sociedad Cooperativa Andaluza en la zona que está implantada (Málaga). Uno de los stakeholders entrevistados nos comenta:

\section{"[...] Málaga es prácticamente Hojiblanca"}

Se puede afirmar que la mayoría de la población de la zona de Antequera (Málaga) está vinculada a esta sociedad cooperativa. De este modo, se puede considerar nuestro caso de estudio como un motor de desarrollo de la localidad, al ser una fuente de ingresos importante que propicia la aparición de una gran actividad comercial ligada a la misma. Este es el discurso de uno de los stakeholders entrevistados:

"Sería muy difícil entender la economía de muchos municipios sin la presencia de las cooperativas"

Por otra parte, para Hojiblanca es básico estrechar vínculos con el entorno para mejorar las relaciones con éste, en particular con las diferentes administraciones. Con esta finalidad establece numerosas relaciones institucionales tanto a nivel nacional y regional, como local y comarcal.

También realiza numerosas colaboraciones con el entorno en el que está inmersa. Se trata de colaboraciones educativas de todos los niveles, pues aporta aceite para desayunos escolares, en fiestas locales y autonómicas. Colaboraciones institucionales con la Consejería de Cultura y la de Agricultura de la Junta de Andalucía, con las Diputaciones Provinciales,..., colaboran con diferentes asociaciones (agrarias, vecinales,...) que solicitan apoyo para las actividades que organizan. En definitiva, se puede comprobar cómo Hojiblanca influye positivamente en el desarrollo de su entorno a cualquier nivel nacional, regional y local. 


\section{CONCLUSIONES Y FUTURAS LÍNEAS DE TRABAJO}

En las últimas décadas, el movimiento cooperativo en todo el mundo intenta resolver un interrogante esencial de primera magnitud: cómo interactuar con la nueva realidad económica, social y tecnológica del entorno sin amenazar los principios cooperativos y sin sacrificar la particularidad de sus objetivos (Agirre, 2001).

Nuevos retos se presentan para las sociedades cooperativas en el futuro que se pueden sintetizar en: la mejora de la dimensión y la competitividad, la adaptación a las nuevas demandas de mercado, el fomento de la educación y la formación, la concentración y organización de la información, la potenciación de procesos de desarrollo territorial y la homogeneización de las condiciones sociales, normativas y financieras que promueven la intercooperación. Teniendo en cuenta esta realidad, los empresarios del medio rural a través de sus sociedades cooperativas han de estar atentos a la solución de los grandes problemas y la satisfacción de las nuevas demandas (Bel y Ausín, 2007).

Como hemos observado en el caso de estudio analizado la identidad cooperativa continúa siendo válida para enfrentarse al reto que supone la globalización de los mercados. Además esta identidad cooperativa basada en la cultura tan específica de este tipo de empresas y sus principios de funcionamiento particulares posibilita que este tipo de empresa pueda lograr una ventaja competitiva sostenible ante las nuevas condiciones del entorno.

Podemos afirmar que los principios cooperativos suponen una característica diferenciadora de este tipo de empresa, en general y de Hojiblanca S. C. A. en particular, y que está característica puede asegurar una gestión excelente adaptada a las nuevas necesidades del entorno globalizado en el que prima el cambio, la rapidez, la innovación, la flexibilidad y el estar dispuesto a reinventar cada actividad productiva o de servicios. Esta empresa ha intentado aplicar un management comprometido que entiende el propósito y los valores cooperativos y que puede utilizarlos para tener éxito y utilizar las diferencias cooperativas como una ventaja competitiva.

De este modo, la finalidad que hemos pretendido es poner de relieve cómo la profundización y el cumplimiento de los principios cooperativos puede ser un instrumento 
para lograr la supervivencia y la competitividad en la empresa cooperativa Hojiblanca S. C. A.

En definitiva, esta empresa cooperativa representa una realidad empresarial de propiedad colectiva y gestión democrática que trata de fomentar la solidaridad, la participación y la corresponsabilidad. Su funcionamiento se rige por los principios cooperativos que constituyen su particularidad y esencia. El cumplimiento de estos principios cooperativos permite que se genere capital social en esta empresa cooperativa, el cual constituye un activo intangible de carácter estratégico que puede influir en la competitividad de la organización. Además, como hemos observado el cumplimiento y la profundización en los principios cooperativos ha supuesto una mejora en la relación entre los stakeholders de la empresa cooperativa, este resultado es acorde con el trabajo de Marcuello y Saz (2008).

Según el último de los principios cooperativos, “al mismo tiempo que se centran en las necesidades y los deseos de los socios, las sociedades cooperativas trabajan para conseguir el desarrollo sostenible de sus comunidades según los criterios aprobados por los socios" (ACI, 1995). Este principio supone, como se ha puesto de manifiesto la búsqueda de la cohesión social con el entorno en el que está inmersa la empresa. Asimismo, como hemos observado en el caso de estudio se ofrece la posibilidad de desarrollar la zona geográfica de referencia en la que se implanta la organización.

Con este trabajo se ha intentado mejorar el conocimiento de la sociedad cooperativa a través del caso de la empresa Hojiblanca S.C.A. Del análisis de este caso, hemos comprobado cómo la aplicación de los principios cooperativos, y en particular el último de ellos, es básico para lograr una ventaja competitiva en la organización y propiciar el desarrollo y cohesión social de la zona de referencia. Se ha observado cómo los principios cooperativos ponen en práctica los valores del cooperativismo a través de la gestión participativa. De este modo, podemos entender el estudio del caso analizado como ilustrativo para futuras investigaciones.

Para finalizar, hemos de precisar que los principios cooperativos tienen una misma función: servir de cauce para forjar una estructura cooperativa eficaz y adecuada a los intereses de todos sus integrantes. Por tanto, creemos que respetando estos principios, la sociedad cooperativa adquirirá plena competitividad en el mercado. 


\section{BIBLIOGRAFÍA}

AECA (2004): Marco conceptual de la responsabilidad social corporativa. Comisión de responsabilidad social corporativa.

AGIRRE, A. (2001). "Los principios cooperativos "atractores" de la gestión eficiente: su medición. Aplicación al caso de Mondragón Corporación Cooperativa”. CIRIEC-España, Revista de Economía Pública, Social y Cooperativa, 39, pp. 93-114.

ALIANZA COOPERATIVA INTERNACIONAL (ACI) (1995): “Los principios cooperativos del siglo XXI". CIRIEC-España, Revista de Economía Pública, Social y Cooperativa, 19, pp. 38-39.

ALIMARKET (2008): "Ranking de las 5000 mayores empresas de España". Publicaciones Alimarket S.A.

ÁlVAREZ, J. (1999): "Priorización de los stakeholders en la actual sociedad del conocimiento". Técnica Contable, 610, pp. 665-670.

ARCAS, N. y RUÍZ, S. (1997): "La comercialización de frutas y hortalizas en el entorno internacional”. Distribución y Consumo, 7 (35), pp. 55-91.

BEL, P. y AUSÍN, J.M. (2007): “Contribución de las sociedades cooperativas al desarrollo territorial". REVESCO, 92(2), pp. 41-71.

BERGLÖF, E. y CLAESSENS, S. (2006): "Enforcement and Good Corporate Governance in Developing Countries and Transition Economies". The World Bank Research Observer, 21 (1), pp. 123-150.

BREALEY, R.A. y MYERS, S.C. (1993): Fundamentos de Financiación Empresarial. Edición Mc Graw-Hill, Madrid.

CABALLERO, G. (2005): Los stakeholders en el modelo de gobierno corporativo. Un análisis del impacto en las estrategias de empleablidad de la universidad española. Tesis Doctoral, Universidad de Vigo.

CCAE (2001): Cooperativismo en cifras. Publicado en www.agro-alimentarias.coop

CHAVES, R. (2004): “Gobierno y democracia en la economía social”. Revista Mediterráneo Económico, 6, pp. 35-52.

CHAVES, R. y SOLER, F. (2004): El gobierno de las cooperativas de crédito en España, CIRIEC-España y Ministerio de Trabajo y Asuntos Sociales, Valencia.

CHEUNG, Y.L.; CONNELLY, Th.; LIMPAPHAYON, P. y ZHOU, L. (2007): “Do Investors Really Value Corporate Governance? Evidence from the Hong Kong Market". Journal of International Financial Management \& Accounting, Oxford; 18 (2), pp.86. 
CORNFORTH, C. (2002): "Making Sense of Co-operative Governance: Competing Models and Tensions". Review of International Co-operation, 95(1), pp. 51-57.

DAVIS, P. (2001): “The governance of co-operatives under competitive conditions: issues, processes and culture". Corporate Governance, 1(4), pp. 28-39.

DÍAZ, F. (2000): “Cooperativismo, valores e ideología”. Anuario de Estudios Cooperativos, 1, pp. $127-142$.

DÍAZ, F. (2004): “Actitudes, motivaciones y creación de empresas: el caso de los emprendedores en sistema cooperativo". REVESCO, 24, pp. 37-52.

EISENHARDT, K. M. (1989): “Agency Theory: An Assessment and Review”. Academy of Management Review, 14(1), pp. 57-74.

FREEMAN, E. (1984): “Strategic Management: A stakeholder approach”. Boston: Printman Press.

GARCÍA, E. (2000): La profesionalización de la sociedad cooperativa almazarera andaluza: un enfoque de agencia. Tesis Doctoral, Universidad de Jaén.

GÓMEZ, P. y MIRANDA, G. (2006): “Sobre el régimen económico y financiero particular de las sociedades cooperativas". REVESCO, 90, pp. 28-56.

GONZÁLEZ, E. (2001): La responsabilidad moral de la empresa: una revisión de la teoría de stakeholder desde la ética discursiva. Tesis doctoral, Universidad Jaume I de Castellón.

GUERRAS, L.A. y NAVAS, J.E. (2007): la Dirección Estratégica de la Empresa. Teoría y Aplicaciones. Thompson Civitas.

HERMES, N.; POSTMA, Th. J.B.M. y ZIVKOV, O. (2006): “Corporate governance codes in the European Union; Are they driven by external or domestic forces?" International Journal of Managerial Finance, 2 (4), pp. 280.

HERNÁNDEZ, M. J. (2001): Base Social de las sociedades cooperativas almazareras. Caja Rural de Jaén.

HERNANDEZ, M. E. y MUNUERA, J. L. (1997): Consideraciones sobre la competitividad de la empresa de comercialización hortofrutícola. Distribución y Consumo, Agosto/Septiembre.

HERNÁNDEZ, M.J.; RUÍZ, C. Y GARCÍA, E. (2008): "La cultura como mecanismo de gobierno y control en las organizaciones cooperativas”. REVESCO, 95, pp. 94-111.

JORDAO, J. y BRIZ, J. (2004): “Aproximación sostenible del tema del cooperativismo en Portugal: situación actual y perspectivas para el futuro". Congreso Internacional del Cooperativismo Agrario y Desarrollo Rural, Valencia. 
JULIÁ, J.F. (1999): "La concentración en el cooperativismo agroalimentario". Cuadernos de Agricultura, Pesca y Alimentación.

LIZCANO, J.L. (2006): "Buen gobierno y responsabilidad social corporativa", Partida Doble, 182, pp.20-35.

MALLIN, CH. (2003): “Los Códigos de Buen Gobierno: la experiencia británica”. Análisis Financiero, 90 (número extraordinario), pp. 73-80.

MARCUELLO, C. y SAZ, M.I. (2008): "Los principios cooperativos facilitadores de la innovación: un modelo teórico". REVESCO, 94, pp. 59-79.

MARTÍNEZ, A. (1990): Análisis de la integración cooperativa. Universidad de Deusto, Bilbao.

MASCAREÑAS, J.M. y LEJARRIAGA, G. (1993): “Análisis de la estructura de capital de la empresa”. Ed. EUDEMA Universidad.

MCGUNABLE, D.M. (2007): “Corporate Governance in China”. The Business Review, Cambridge, 8 (1), pp. 52-60.

MENGUZZATO, M. y RENAU, J.J. (1991):“Innovación Tecnológica, hilera de producción y estructura de una industria”. Esic market, 73, pp. 93-102.

MITCHELL, R.; AGLE, B. y WOOD, D. (1997): “Toward a theory stakeholder identification and salience: defining the principle of who and what really counts". Academy of Management Review, 22(4), pp. 853-886.

MOREIRA, M.A. (1999): “Globalizacao e o movimento cooperativo. Tópicos para o debate”. En O Cooperativismo no novo milenio. A vantagem cooperativa. Lisboa: INSCOOP, pp. 53-94.

NILSSON, J. (1996): “The nature of cooperative values and principles". Annals of Public and Cooperative Economics, 67(4), pp. 633-653.

OCDE (1999 y 2004): Principios de Gobierno Corporativo

OKIKE, E.N.M. (2007): "Corporate Governance in Nigeria: the status quo". Corporate Governance, 15 (2), pp. 173.

PASS, CH. (2006): "The revised Combined Code and corporate governance: An empirical survey of 50 large UK companies”. Managerial Law; 48 (5), pp. 467-478.

PEDROSA, C. (2009): Factores de éxito en la gestión de las sociedades cooperativas: el caso de Hojiblanca. Trabajo de Investigación realizado en el Departamento de Organización de Empresas, Marketing y Sociología de la Universidad de Jaén.

REVESCO No 106 - Tercer Cuatrimestre 2011 - ISSN: 1885-8031 - www.ucm.es/info/revesco 
PEDROSA, C. (2009): “Modelos teóricos que nos ayudan a comprender el gobierno de las sociedades cooperativas, una apuesta por el enfoque de los stakeholders”, Revista de la Agrupación Joven Iberoamericana de Contabilidad y Administración de Empresas, 4, pp. 54-71.

PLANELLS, J.M. y MIR, J. (2000): “Situación actual de la Distribución Europea”. Levante Agrícola, Primer trimestre, pp. 76-85.

REAZ, M. y HOSSAIN, M. (2007): "Corporate Governance around the World: An Investigation". Journal of American Academy of Business, Cambridge, 11 (2), pp.169175.

ROMERO, A.J. y PÉREZ, M., (2003): “Organizational culture, individual differences and the participation system in cooperativism of associated workers in Andalusia, Spain". Annals of Public and Cooperative Economics, 74(2), pp. 283-320.

RUÍZ, M.C.; HERNÁNDEZ, M.J. y GARCÍA, E. (2008): “La identidad cooperativa como respuesta al desafío de la globalización: la búsqueda de la excelencia empresarial”. 27 Congreso Internacional de CIRIEC (Sevilla, 22-24 Septiembre).

RYAN, B.; SCAPENS, W., y THEOBAL, M. (1992): "Research Method and Methodology in Finance and Accounting”, Academic Press.

SCOTT, S. y LANE, V. (2000): “A stakeholder approach to organizational identity". Academy of Management Review, 25(1), pp. 43-62.

SPEAR, R. (2004). "El gobierno democrático en las empresas cooperativas", CIRIECEspaña, Revista de Economía Pública, Social y Cooperativa, 48, pp. 11-30.

TALIENTO, M. (2007): "The Role and The Ambit of Corporate Governance and Risk Control Frames". Journal of American Academy of Business, Cambridge, 11 (2), pp. 251256.

TIPURIC, D., HRUSKA, D. y ALEKSIC, A. (2007): “Corporate Governance and Ownership Concentration in Croatia". The Business Review, Cambridge, 7 (1), pp. 207-211.

TOUS, D. y CIRUELA, A.M. (2005): "Responsabilidad social y cultura en las sociedades cooperativas agrarias. Modelo general de balance social”. CIRIEC-España, Revista de Economía Pública, Social y Cooperativa, 53, pp. 209-240.

VARGAS, A. (1995): "La identidad cooperativa y la cooperativa como empresa: luces y sombras". REVESCO, 61, pp. 179-192.

VARGAS, A. (1999): "De la participación en la empresa a la empresa de participación democrática”. REVESCO, 67, pp. 219-234. 
VILLARREAL, O. y LANDETA, J. (2007): "El estudio de casos como metodología de investigación científica en economía de la empresa y dirección estratégica". XXI Congreso Anual EADEM, Universidad Rey Juan Carlos, Madrid, 6-8 Junio.

VON ROSEN, R. (2007): “Corporate Governance in Germany”. Journal of Financial Regulation and Compliance, 15 (1), pp. 30.

WHEELER, D. y SILLANPAA, M. (1998): "Including the stakeholders: the business case". International Journal of Strategic Management: Long Range Planing, 31(2), pp. 201-210.

YIN, R. K. (1984/1989): Case Study Research: Design and Methods, Applied social research Methods Series, Newbury Park CA, Sage. 